\title{
Agent-Based Simulation to Assess the Performance of Intersections with Pre-Signals: Comparison with Roundabouts
}

\author{
António A.C. Vieira, Luís M.S. Dias, Guilherme, A.B. Pereira, José A. Oliveira \\ University of Minho, Campus Gualtar, 4710-057, Braga, Portugal, \\ University of Minho, Portugal \\ \{antonio.vieira, 1sd,gui,zan\}@dps.uminho.pt
}

\begin{abstract}
Solving traffic congestion problems in intersections is a complex logistic problem that usually consists in building infrastructures, such as bridges, tunnels, or roundabouts, which represent the costliest solutions. Concerning the case of roundabouts, many parameters influence its performance, e.g. geometry and size. Thus, other less costlier solutions should be pondered. This paper introduces a low-cost approach to traffic intersections, by using pre-signals; and conducts simulation experiments to verify if this approach could be used to improve the performance of traffic intersections. In this sense, an agent-based traffic simulation model was developed that applies the object modelling paradigm of Simio to model the individual behaviour of vehicles. The simulation experiments results indicate that the flow of vehicles can be increased up to $20 \%$, reducing the average queue sizes and crossing time per vehicle and saving the fuel consumed up to $64 \%$.
\end{abstract}

\section{Introduction}

Since the motor vehicle became the main means of transport, many traffic congestion problems can be witnessed. To overcome them, usually two types of solutions are used: optimization approaches for the duration of traffic light phases, or physical-changes (Heng and Perugu, 2009), such as the expansion of the intersection, or the construction of tunnels, bridges or roundabouts, representing a more onerous type of solution (Treiber and Helbing, 2001).

In a previous work, Vieira et al. (2014b) presented the pre-signals concept as a lowcost technique to improve the performance of an intersection and compared an intersection implementing this concept with a regular intersection. The concept consists on implementing an additional traffic-light on each lane, located prior to the main traffic-light of each lane. To implement them, it is necessary to provide some dozens of meters on the approaches of the intersection, working as "launch pads". The achieved results indicated that the best values to use for the green signal on intersections with pre-signals (different from the best green signal duration on normal intersections) and the best distance to keep between a pre-signals and its main traffic-light: 20 seconds of green signal, at about 40 meters of distance to the pre-signals, are enough to allow 
maximum flow and minimize queue sizes and waiting time per vehicle. These values will be used in the work presented in this paper.

This paper presents a discrete-event simulation model, developed to compare the performance of intersection with pre-signals and roundabouts. It was developed in Simio, a recently developed object oriented discrete simulation tool that also supports the agent modelling approach and other approaches, such as processes and events.

Simulation enables the visualization of the results from modifications made to a system, without making experiments in the real world. To address this kind of traffic-related problems, usually traffic simulation packages, such as VISSIM are used. However, to the best of the knowledge of the authors, these traffic simulation packages lack the ability of modelling concepts not standardized, such as the one here proposed. As such, discrete-event simulation was used for this work.

Next section reviews the literature on this topic. Section 3 is dedicated to the data gathering and validation processes. In section 4 , the main tasks conducted to develop the simulation model will be covered and section 5 is related to the simulation experiments conducted. Conclusions are discussed in the last section.

\section{Literature Review}

Despite only having been first documented in 1991 in the UK (Oakes et al., 1994), presignals were already in use in several European cities (Wu and Hounsell, 1998). Its implementation is becoming significant in some cities of the United Kingdom and, in fact, until 1993, only in London, 14 pre-signals were implemented and a further 20 to 25 pre-signals were planned for the coming years (Wu and Hounsell, 1998).

The implementation of pre-signals can have many goals. One of these is "to give buses priority access into a bus advance area of the main junction stop line so as to avoid the traffic queue and reduce bus delay at the signal controlled junction" (Wu and Hounsell, 1998). Conversely, in Xie and Ma (2012) pre-signals were used to avoid losses of capacity on the lanes that cannot discharge completely during its green phases, due to the existence of turning lanes. More recently, Xuan et al. (2011) were pioneers on the utilization of pre-signals to increase the capacity of a traffic intersection. In their study the approaches receives " 2 green sub-phases: one for protected left turns only, and the other exclusively for through movements and right turns" (Xuan et al., 2011). In Zhou and Zhuang (2013), this idea was seized and the authors proposed "an integrated model for lane assignment and signal timing optimization at tandem intersections". The model aimed to minimize the average delay that vehicles experienced in the pre-signal and main signal.

Currently there are not many studies that use Simio for modelling traffic related problems. A possible justification for this is that most of the studies that use simulation in problems related to traffic, use packages of micro simulation tools like VISSIM or AIMSUN. However, to test the applicability of new proposed concepts such as the one proposed in this paper, to the best of the knowledge of the authors, these simulation packages reveal to be inadequate, since they are prepared to model only certain infrastructures types. Therefore, for this study, a discrete event simulation software was 
used. The number of commercial tool options can be very high, thus simulation tool comparison becomes a very important task.

In Hlupic and Paul (1999), a set of simulation tools were compared, distinguishing between users of software for educational purposes and users in industry. In his turn, Hlupic (2000) developed a survey of academic and industrial users on the use of simulation software, to discover how the users are satisfied with the simulation software they use and how this software could be further improved. In Dias et al. (2007), Pereira et al. (2011) and Dias et al. (2016) a comparison of tools based on popularity on the internet, scientific publications, WSC (Winter Simulation Conference), social networks and other sources, was established. According to the authors, popularity should not be used as the only comparison indicator, otherwise new tools, better than existing ones, would never get market place. However, a positive correlation may exist between popularity and quality, since the best tools have a higher chance of being more popular. According to this ranking, the most popular tool is Arena, whilst the classification of the "newcomer" Simio is noteworthy. Vieira et al. (2014a) and Oueida et al. (2016) compared both tools taking into consideration several factors.

Simio was created in 2007 from the same developers of Arena and is based on intelligent objects (Sturrock and Pegden, 2010, Pegden, 2007, Pegden and Sturrock, 2008). In this tool, a vehicle, a costumer or any other agent of a system are examples of possible objects and, combining several of these, one can represent the components of the system in analysis. In other words, the user can use realistic representations of the objects that compose the real system being modelled and, thereafter, at a lower level, define additional logic to the model, through the development of processes for instance. Thus, in Simio the model logic and animation are built in a single step (Pegden and Sturrock, 2008, Pegden, 2007), making the modelling process very intuitive with the addition of a full built-in 3D animation.

\section{Data Collection and Validation}

In this section, some of the data that was gathered and entered into the developed model will be presented and explained. It should be noted that, some of these data had already been explained in Vieira et al. (2014b), namely those related to the intersection with pre-signals. To build a model capable of representing the real system, the following data related traffic situations was gathered through literature collected and analysed:

- Cycle times of the traffic lights: Pan et al. (2010) stated that when the signal cycle length is around 100 seconds, the waiting time of vehicles is minimal.

- Safety distances kept while driving: Drivers that travel at a speed next to $50 \mathrm{~km} / \mathrm{h}$ maintain a safety distance of about 16 meters (Luo et al., 2011).

- Space occupied by a vehicle in a queue: The analysed studies indicate that a stopped vehicle occupies a distance between 7.6 meters and 7.9 meters (Bonneson, 1992, Messer and Fambro, 1997, Zhu, 2008, Herman et al., 1971)

- Start-up acceleration: Zhu (2008) analysed several studies regarding this matter. The author developed a polynomial acceleration model characterized by expression (1). Since in Simio it is not possible to implement the acceleration of entities, it was necessary to use the correspondent velocity expression (2). 
$a=2,46-0,24 t+0,006 t^{2}$

$v=2,66+2,46 t-0,12 t^{2}+0,002 t^{3}$

- Time the drivers on the first position of a queue take to react to a traffic light signal change to green: Some authors considered that the first vehicle of a queue normally wastes 2 seconds to initiate the start-up acceleration process after the traffic light changes to green (Bonneson, 1992, Messer and Fambro, 1997); others, considered the wasted time lies between 1.5 and 2 seconds (Bonneson, 1992, George and Heroy, 1966).

- Time spent, by the drivers on the remaining positions of a queue, to react to a traffic light signal change to green: According to Bonneson (1992), these values correspond to 1 second per vehicle, 1.22 seconds or 1.3 seconds.

- Reaction time of drivers on roundabouts: It is difficult to find in the literature and to measure in the filed the reaction time that drivers take to start accelerating, from a resting position, in a roundabout queue. This is because drivers are constantly trying to access a gap in the roundabout and many times they do not completely stop, which influences their start-up accelerating process. This does not happen, for instance, in signalized intersection, since drivers must wait for a red light that they do not know when it is going to change. Thus, the reaction time of drivers in the queues of signalized intersection was used. As already stated, these values can go from 1 to 1.3 seconds for the vehicles in the first position of the queue, to 1.5 to 2 seconds for the remaining positions. These values were incorporated in Simio, adjusting them to have the reaction time of drivers being dependent on their distance to the one on the first position of the queue, as authors agree. Moreover, since the reaction time of drivers in roundabouts is lower than on signalized intersections, these values were calibrated. Thus, in this model, the first vehicle of the queue on the signalized intersection took considerable more time than the vehicle on the same position of the queue of the roundabout. Concerning the reaction time of the vehicles on the remaining positions, their values decrease until an average of 1 second. After that, the average value is maintained.

- Velocity while circulating inside the roundabout: Skrodenis et al. (2011) stated that speeds of vehicles, circulating inside roundabouts, of diameter varying between 16 to 45 meters, should be around $16-30 \mathrm{~km} / \mathrm{h}$. Furthermore, the speed of vehicles entering and circulating roundabouts tends to be higher for bigger roundabouts (Brilon, 2005). Based on this and on numerous calibrations to the simulation model, it was considered that the vehicles could accelerate to a maximum speed of $30 \mathrm{~km} / \mathrm{h}$ in roundabouts of similar size. For smaller roundabouts, the vehicles will only be able to speed up until $25 \mathrm{~km} / \mathrm{h}$. While circulating on roundabouts of 60 meters of diameter the vehicles will be able to speed up until $35 \mathrm{~km} / \mathrm{h}$ and on roundabouts of 80 meters the vehicles will be able to speed up until $40 \mathrm{~km} / \mathrm{h}$. Thus, these speed differences also have an influence on the space gap required by the drivers to access the roundabouts of different sizes.

- Space gap to access the roundabout: While circulating a roundabout, the velocity of a vehicle affects the required space, or time, for a second vehicle to access the same roundabout. Since these values were modelled based on data collected from the literature, the authors empirically calibrated the required space gap, in order to 
minimize the occasions on which a vehicle decides to access a roundabout and, because of that, another vehicle, circulating on the roundabout, had to slowdown, since the available gap was too small for the other vehicle to access the roundabout. Thus, the space required for a vehicle, to access the roundabout was 17 meters for the roundabout of around 10 meters of radius, 22 meters for the roundabout with around 20 meters of radius, 33 meters for the roundabout of a radius of around 30 meters and 47 meters for the roundabout with around 40 meters of radius.

- Discharge rate of a queue: Bonneson (1992) concluded that this takes place at a rate of approximately 1 vehicle per 2 seconds. Other authors considered lower values like 1.97 (Lee and Chen, 1986) or 1.92 seconds (Zegeer, 1986).

- Instant speed when crossing the stop line of an intersection: Bonneson (1992) stated that the velocity of each vehicle increases until the fourth or fifth vehicle. From that number, the velocity of the vehicles tends to stabilize.

- Fuel consumption and emission rates: Some of the models that estimate consumption rates and emissions include those based on the instant velocity of vehicles. Tong et al. (2000) established a formula for the fuel consumption of diesel vehicles in order of the instantaneous vehicle speed, whilst Chan et al. (2004) used a formula to estimate "the fuel consumption of petrol vehicles as a function of the instantaneous vehicle speed". Notwithstanding, there are models that consider other factors, such as the model proposed by Akçelik and Besley (2003), which considers the acceleration of the vehicle, its mass, instant speed, among other parameters. Akçelik (1983) also provided a model that expresses fuel consumption as a function of cruising, idling and stop-start manoeuvers. In its turn, Guo and Zhang (2014) indicated the formula currently being used by some traffic micro simulation tools (c.f. VISSIM, TRANSYT, and SYNCHRO).

Apart from formulas that estimate the consumption and emission rates, Coelho et al. presented the emission factor of $\mathrm{HC}, \mathrm{NOx}, \mathrm{CO} 2$ and $\mathrm{CO}$ for several vehicle speed powers (2006). In its turn, Tong et al. collected data related to vehicle speed, emission, and fuel consumption from four types of vehicles while they travel on different driving modes (i.e., idle, acceleration, cruise and deceleration) (2000). The authors presented the results in $\mathrm{g} / \mathrm{km}, \mathrm{g} / \mathrm{sec}$ and $\mathrm{g} / \mathrm{kg}$ fuel. Even though, there are more recent works that provide similar data, like the one Lau et al. (2011) conducted. These authors studied the $\mathrm{CO}, \mathrm{NO}$ and $\mathrm{HC}$ emission rates, as well as the fuel consumption rates from four LPG taxis of different years, driven under urban traffic conditions. Notwithstanding, the data used in this study was the one collected by Tong et al. (2000), since it considers the time the drivers spend on each of the four driving modes. Thus, is consists on a simple, yet efficient, way to model the main consume patterns. The data provided by the authors and used on this study is presented in Table 1. Despite its age, to the best of the knowledge of the authors, this reference was the only one we could find meeting the previously stated established requirements. Nowadays, all these values should be inferior, albeit at the same proportion. 
Table 1. Modal emission and fuel consumption rates (Tong et al., 2000)

\begin{tabular}{llllll}
\hline \multirow{4}{*}{ Passenger Car } & Driving mode & \multicolumn{2}{l}{ Modal emission rate $(\mathrm{mg} / \mathrm{sec})$} & Fuel Consumption \\
& & CO & HC & NOx & \\
\hline \multirow{5}{*}{ Petrol Van } & Acceleration & 9.54 & 0.69 & 0.62 & 62.62 \\
& Cruising & 9.15 & 0.49 & 0.77 & 39.1 \\
& Deceleration & 9.96 & 0.58 & 0.69 & 28.11 \\
& Idling & 2.99 & 0.36 & 0.14 & 18.11 \\
& Acceleration & 15.14 & 1.85 & 1.96 & 67.29 \\
& Cruising & 14.52 & 1.70 & 1.81 & 52.14 \\
& Deceleration & 17.30 & 1.91 & 2.33 & 52.16 \\
& Idling & 8.39 & 1.88 & 0.81 & 12.71 \\
& Acceleration & 2.71 & 0.65 & 0.91 & 62.02 \\
& Cruising & 2.64 & 0.54 & 0.79 & 52.47 \\
& Deceleration & 2.67 & 0.65 & 0.89 & 56.01 \\
& Idling & 1.33 & 0.22 & 0.44 & 18.52 \\
\hline
\end{tabular}

\section{Model Development}

To provide greater realism to the model, 3D models of road segments, traffic lights, vehicles and safety cancels were downloaded from Google Warehouse. Some sample videos of the model in execution were recorded and can be watched online at the following address: http://pessoais.dps.uminho.pt/lsd/pre_semaforos/. Fig. 1 illustrates the developed simulation model modelling a roundabout. In signalized intersection, signal cycles are processed on a counter clockwise direction, through the regular repetition of green, yellow and red lights.

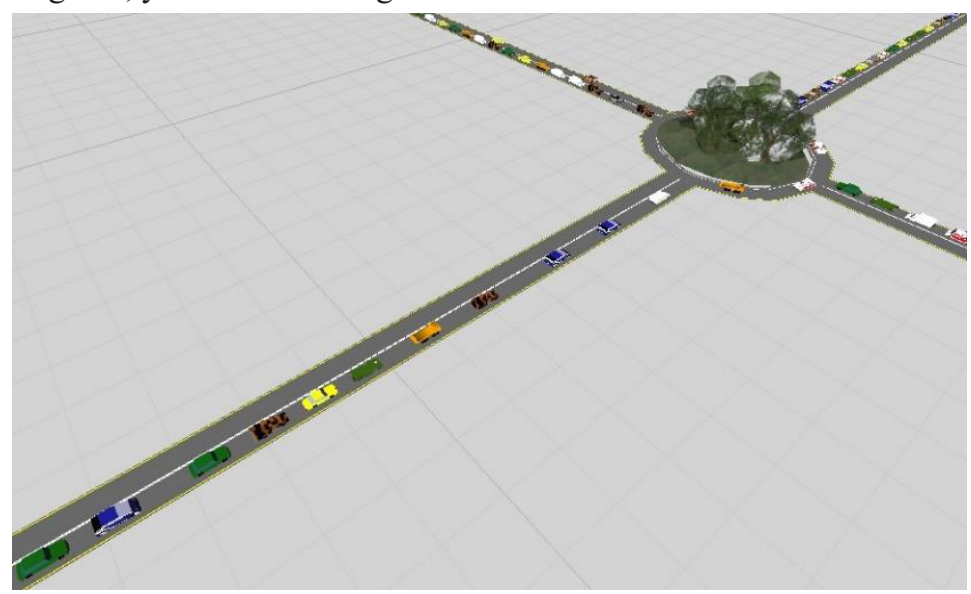

Fig. 1. 3D view of the modelled roundabout

The main processes of the model of the signalized intersection were already described in a previous paper (Vieira et al., 2014b). To access the roundabouts, each vehicle is dynamically executing several processes. In one of those processes, each entity is actively deciding - agent modelling - if it can enter or not the roundabout, by analysing 
the distance to the closest cars at his left, on the roundabout. This decision depends on the size of the roundabout, since for smaller roundabouts the vehicle may have to analyse the number of vehicles traveling the roundabout and approaching its entry lane and even the two previous entry lanes; conversely, for bigger roundabouts, each vehicle may only need to evaluate part of the roundabout between its entry lane and the previous one. Apart from this, the characteristics of the drivers also influences the decision of entering the roundabout, since more cautious drivers require a bigger gap than less cautious do. This process is also responsible for modelling these and other decisions related to the entry of in the roundabout, as well as the acceleration of vehicles that decide to enter the roundabout. Moreover, this process is also responsible for adjusting the speed of vehicles circulating inside the roundabout, depending on the size of the roundabout.

To model the behaviour of the vehicles, both on an intersection with traffic lights or on roundabouts, it was necessary to create many processes, functions, states among others, on the Simio software, to model all the traffic situations, e.g. to maintain a safety distance between vehicles. Nonetheless, in this paper, only some of the processes will be illustrated. Fig. 2 shows the process responsible for updating the fuel and emissions rates of the vehicles. To accurately calculate these rates, the 4 distinct operating modes of the vehicles (i.e. idle, acceleration, cruise and deceleration) had to be correctly defined.

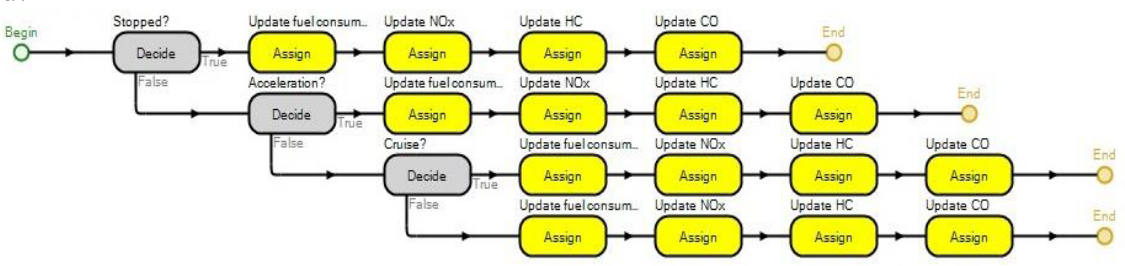

Fig. 2. Process to update consumption and emissions

\section{Simulation Experiments}

In this section, a comparison between the results obtained by modelling an intersection with pre-signals and roundabouts will be established. For the present work, the authors considered the following properties, or parameters, for the conducted simulation experiments:

- the frequency with which the vehicles arrive to the system,

- the radius of the roundabout,

- the balancing of the roundabout,

- the different driving behaviour pattern;

- and the type of intersection, i.e., roundabout or intersection with pre-signals.

As KPI (Key Performance Indicators), the following were defined:

- KPI1: The average flow of vehicles in vehicles/hour. This KPI is the inverse of the time interval between passages of vehicles through the intersection; 
- KPI2: Average crossing time per vehicle in seconds. This KPI is calculated considering the elapsed time when a vehicle is created and when it travels an additional distance of 150 meters after having crossed the intersection;

- KPI3: The average number of vehicles on the queues. This KPI is measured every minute;

- KPI4 to KPI7: The average total fuel consumed per vehicle in milligrams and the average total emissions of vehicles in milligrams (CO, HC and NOx). KPI 4 to KPI 7 respectively refer to fuel consumed, $\mathrm{CO}, \mathrm{HC}$ and NOx emissions. These KPI start being accounted when vehicles are created and they are updated every minute. When a vehicle crosses the intersection, these KPI are recorded;

- KPI8: the average number of stops per vehicle.

Moreover, the values 4, 8, 13 and 50 seconds were considered, respectively, for the time interval that defines the creation of vehicles, i.e., the intensities very high, high, medium and low. Based on previous results (Vieira et al., 2014b), a warm-period of 360 seconds was used, along with a simulation time of 2 hours and 6 replications.

Regarding the roundabouts that were modelled, it was considered that only $50 \%$ of the drivers that are trying to access a roundabout, do it, when they notice a vehicle, circulating in the roundabout signalizes its exit in the next lane. This also covers situation in which the driver circulating inside the roundabout does not signalize its intention to leave in the next exit, forcing the vehicle that is trying to access the roundabout to wait. It should be noted that this percentage can be adjusted. In addition, vehicles do not choose the exit of a roundabout in equal proportions, i.e., these exit lanes have different weights on the overall capacity of the roundabout. Considering this, different weights were assigned to the 4 lanes of the modelled roundabouts respectively $40 \%, 30 \%, 20 \%$ and $10 \%$.

Fig. 3 shows the values obtained for the flow of vehicles of 2 types of roundabouts, under very high traffic conditions. The 2 compared roundabouts consist on an optimistic one and the one that was modelled with the purpose of conducting the simulation experiments for this study. The difference between these types of roundabouts is that the former does not consider what was exposed, regarding the weights of the exits of roundabouts and the different driving behaviour, and the latter considers.

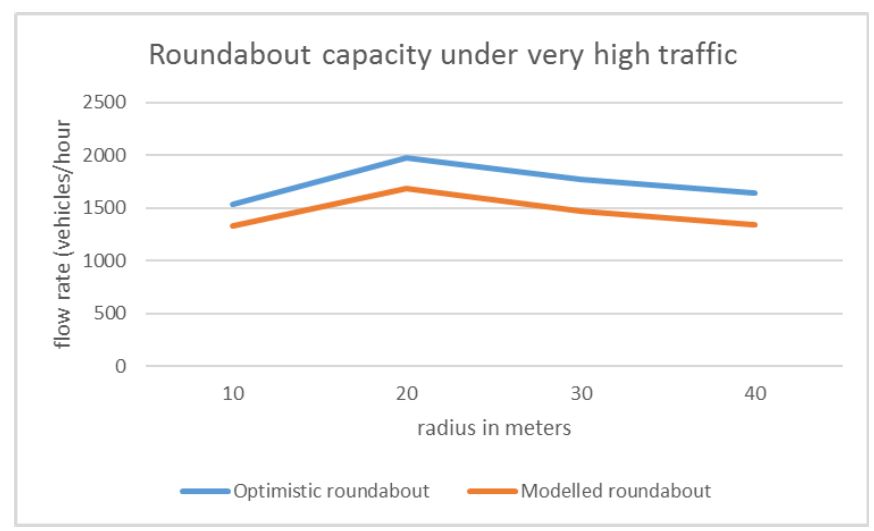

Fig. 3. Flow rates of roundabouts modelled with different sizes 
As can be seen, regardless of the type of roundabout the best size to be used would be around 40 meters of diameter, which was also found by Oketch et al. (2004). It can also be noted that the different weights assigned to the roundabout and the different driving behaviour impact the overall performance of the system. Table 2 summarizes the results to be discussed.

Table 2. Comparing all the modelled intersections

\begin{tabular}{cccccccccc}
\hline $\begin{array}{c}\text { Traffic } \\
\text { intensities }\end{array}$ & $\begin{array}{c}\text { Intersection } \\
\text { type }\end{array}$ & KPI1 & KPI2 & KPI3 & KPI4 & KPI5 & KPI6 & KPI7 & KPI8 \\
\hline \multirow{2}{*}{ Very high } & Roundabout & 1686 & 9,19 & 63,63 & 16,71 & 2,72 & 0,25 & 0,16 & 32,63 \\
& Pre-signals & 2106 & 7,15 & 67,89 & 8,97 & 1,68 & 0,17 & 0,09 & 5,12 \\
\multirow{5}{*}{ High } & Roundabout & 1650 & 4,5 & 26,57 & 8,62 & 1,51 & 0,12 & 0,1 & 14,91 \\
& Pre-signals & 1801 & 1,49 & 9,35 & 3,01 & 0,62 & 0,04 & 0,05 & 0,9 \\
& Roundabout & 1108 & 1,1 & 0,15 & 2,69 & 0,61 & 0,03 & 0,05 & 0,39 \\
\multirow{2}{*}{ Low } & Pre-signals & 1109 & 1,44 & 5,61 & 2,9 & 0,61 & 0,04 & 0,05 & 0,82 \\
& Roundabout & 289 & 1,07 & 0,01 & 2,63 & 0,6 & 0,03 & 0,05 & 0,19 \\
& Pre-signals & 288 & 1,42 & 1,5 & 2,84 & 0,6 & 0,04 & 0,05 & 0,75 \\
\hline
\end{tabular}

As the simulation experiments results illustrate, for low and medium traffic intensities, both modelled roundabouts obtained the best results for all the KPI, aside from the average flow of vehicles, when compared to the remaining three modelled intersections. Thus, on these traffic intensities, roundabouts achieved a better performance than the modelled intersection with pre-signals, as has been previously observed (Fouladvand et al., 2003, Skrodenis et al., 2011). These observations can be explained by the fact that, on a roundabout, the vehicles only wait for a gap to access the roundabout, while on an intersection the vehicles must wait for the traffic-light to turn to green. Thus, the vehicles on the roundabout could stop less times and consequently form shorter queues, consume less fuel and emit less polluting gases. Conversely, as the traffic conditions become more saturated, the available gaps on the roundabout become less frequent. Focusing the analysis on the high and very high intensities, it is possible to note that what was observed in the low and medium intensities no longer applies. The only KPI that still got better results in these traffic conditions was the queue size. In fact, it is possible to verify that the realistic roundabout achieved the worse performance in these traffic conditions, for all the KPI, except for the queues size in the very high traffic intensity. In its turn, for the highest traffic intensity, the intersection with pre-signals achieved the best performance for all KPI, except for the queues size and the average number of stops. Thus, on more saturated traffic conditions, the intersection with presignals presented better simulation results than the modelled roundabouts, which is in accordance to previous studies (Fouladvand et al., 2003, Skrodenis et al., 2011).

Focusing the comparison between the realistic roundabout and the intersection with pre-signals, it can be observed that, once again, their performance is not differentiated in low and medium traffic intensities. Regarding the remaining intensities, significative differences can be observed in all KPI. Considering this flow of vehicles KPI, it could be increased from 8 to $20 \%$, representing a difference of roughly 150 to 420 vehicles/hour. Therefore, the intersection with pre-signals could have more vehicles crossing it at the same time, which culminated in significative reduced queues and crossing time per vehicle. In its turn, vehicles stop considerably less times to cross the 
intersection. This is explained by the fact that in most situations vehicles can cross the intersection with just one stop, rather than having to repeatedly stop and start while trying to reach the roundabout and attempting to access it. This culminates with significative differences in the fuel and emissions per vehicle. In fact, vehicles modelled in the intersection with pre-signals spent less 54 to $65 \%$ of fuel than the ones modelled in the roundabout.

\section{Conclusions}

The resolution of traffic congestion problems usually implies the construction of expensive infrastructures such as bridges, tunnels and roundabouts. This paper presented a low-cost solution for this problem that consists on using an additional set of traffic-lights situated some meters away from these, working as pre-signals and acting as "launch-pads" for vehicles in intersection lanes. Thus, a simulation model was developed to evaluate the proposed alternative. In a previous study, this new concept had already been compared to regular signalized intersection with good results (Vieira et al., 2014b). Thus, the second objective of this paper was to compare this new concept to the performance of roundabouts, one of the more expensive type of solutions that is usually adopted, since it involves the construction of an infrastructure and usually much space available.

The first set of conducted simulation experiments focused on evaluating the size of the roundabouts in its performance. It was found that the best size is a diameter of 40 meters. Thereafter, the human factor in the driving behaviour and the unbalancing of the roundabout were considered in its performance. Thus, a realistic roundabout considering its unbalancing and the driving behaviour - and an optimistic roundabout were compared. The main conclusions from this analysis were that the human factor had more negative impact in the performance than the balancing did. In addition, in the highest traffic intensities, the flow of vehicles decreased 8 to $15 \%$ when the optimistic roundabout was compared to the realistic one. It was also found that the unbalancing of roundabouts and the human driving style can decrease the waiting time per vehicle in 3 minutes, the queue size in up to $90 \%$ and the number of stops per vehicle in up to $88 \%$, culminating in an increase in the fuel consumption in up to $63 \%$.

The second set of experiments focused the analysis on the roundabouts and on the intersection with pre-signals. The results were accordingly to previous studies, stating that on low traffic volumes the modelled roundabouts presented better results, while on high intensities the signalized intersection presented better results (Fouladvand et al., 2003, Skrodenis et al., 2011). Focusing the comparison on the highest traffic intensities and comparing the realistic roundabout to the intersection with pre-signals, it was possible to note an increase in the flow of vehicles from 8 to $20 \%$, representing a difference of roughly 150 to 420 vehicles/hour. This was accomplished while still reducing the queues and crossing time per vehicle, which culminated in the vehicles modelled in the intersection with pre-signals spending less 54 to $65 \%$ of fuel than the ones modelled in the roundabout.

Regardless of the positive simulation results achieved, it is still imperial to evaluate the safety of this kind of solution. For future development: (1) it would be interesting to 
adapt the developed traffic model to handle intersections and roundabouts with multi lanes on the approaches, as well as inside the roundabout; (2) illustrate the behaviour of intersections with pre-signals in a context of proximity with other intersections; (3) since agents are being modelled, it would be interesting to model different types of drivers - accelerate more or less, needs more or less space to enter the roundabout, among others; (4) compare the proposed solution to other types of infrastructures, such as bridges and tunnels.

\section{References}

AKÇELIK, R. 1983. Progress in Fuel Consumption Modelling for Urban Traffic Management. Australian Road Research Board.

AKÇELIK, R. \& BESLEY, M. 2003. Operating cost, fuel consumption, and emission models in aaSIDRA and aaMOTION. 25th Conference of Australian Institutes of Transport Research (CAITR 2003).

BONNESON, J. A. 1992. Modeling Queued Driver Behavior at Signalized Junctions. Transportation Research Record, No 1365, pp. 99-107.

BRILON, W. 2005. Roundabouts: A state of the art in Germany. National Roundabout Conference, Vail, Colorado, vol. 16.

CHAN, T. L., NING, Z., LEUNG, C. W., CHEUNG, C. S., HUNG, W. T. \& DONG, G. 2004. On-road remote sensing of petrol vehicle emissions measurement and emission factors estimation in Hong Kong. Atmospheric Environment, 38, 2055-2066.

COELHO, M. C., FARIAS, T. L. \& ROUPHAIL, N. M. 2006. Effect of roundabout operations on pollutant emissions. Transportation Research Part D: Transport and Environment, 11, 333-343.

DIAS, L., PEREIRA, G. \& RODRIGUES, G. 2007. A Shortlist of the Most Popular Discrete Simulation Tools. Simulation News Europe, 17, 33-36.

DIAS, L., PEREIRA, G., VIK, P. \& OLIVEIRA, J. A. 2011. Discrete simulation tools ranking: a commercial software packages comparison based on popularity. Industrial Simulation Conference. Venice, Italy, 6-8 June: Eurosis.

DIAS, L. M. S., VIEIRA, A. A. C., PEREIRA, G. A. B. \& OLIVEIRA, J. A. 2016. Discrete Simulation Software Ranking - a Top list of the Worldwide most Popular and Used Tools. Proceedings of the 2016 Winter Simulation Conference.

FOULADVAND, M. E., SADJADI, Z. \& SHAEBANI, M. R. 2003. Characteristics of vehicular traffic flow at a roundabout. Physical Review E 70.4 (2004): 046132.

GEORGE, E. T. \& HEROY, F. M. 1966. Starting Response of Traffic at Signalized Intersections. Traffic Engineering 36.1Q, pp. 39-43.

GUO, R. \& ZHANG, Y. 2014. Exploration of correlation between environmental factors and mobility at signalized intersections. Transportation Research Part D: Transport and Environment, 32, 24-34.

HENG, W. \& PERUGU, H. C. 2009. Oversaturation inherence and traffic diversion effect at urban intersections through simulation. Jiaotong Yunshu Xitong 
Gongcheng Yu Xinxi/ Journal of Transportation Systems Engineering and Information Technology, 9, 72-82.

HERMAN, R., LAM, T. \& ROTHERY, R. W. 1971. The Starting Characteristics of Automobile Platoons. Proc., 5th International Symposium on the Theory of Traffic Flow and Transportation, American Elsevier Publishing Co., New York, pp. 1-17.

HLUPIC, V. 2000. Simulation software: an Operational Research Society survey of academic and industrial users. Proceedings of the 32nd Winter Simulation Conference (pp. 1676-1683 vol. 2). Society for Computer Simulation International.

HLUPIC, V. \& PAUL, R. 1999. Guidelines for selection of manufacturing simulation software. IIE Transactions, 31, 21-29.

LAU, J., HUNG, W. T. \& CHEUNG, C. S. 2011. On-board gaseous emissions of LPG taxis and estimation of taxi fleet emissions. Science of The Total Environment, 409, 5292-5300.

LEE, J. \& CHEN, R. L. 1986. Entering Headway at Signalized Intersections in a Small Metropolitan Area. Transportation Research Record (1091).

LUO, Q., XUN, L., CAO, Z. \& HUANG, Y. 2011. Simulation analysis and study on car-following safety distance model based on braking process of leading vehicle. 9th World Congress on Intelligent Control and Automation (WCICA). pp. 740-743. IEEE.

MESSER, C. J. \& FAMBRO, D. B. 1997. Effects of Signal Phasing and Length of Left Turn Bay on Capacity, No. 644. 1977.

OAKES, J., THELLMANN, A. M. \& KELLY, I. T. 1994. Innovative bus priority measures. Proceedings of Seminar J, Traffic Management and Road Safety, 22nd PTRC European Transport Summer Annual Meeting, University of WARWICK, U.K., vol.381, pp.301-312.

OKETCH, T., MIKE DELSEY, AND DOUG ROBERTSON. 2004. Evaluation of Performance of Modern Roundabouts Using Paramics Microsimulation Model. TAC Conference.

OUEIDA, S., CHAR, P. A., KADRY, S. \& IONESCU, S. 2016. Simulation Models for Enhancing the Health Care Systems. FAIMA Business \& Management Journal, $4,5$.

PAN, M., DONG, S., SUN, J. \& LI, K. 2010. Microscopic Simulation Research on Signal Cycle Length of Mixed Traffic Considering Violation. International Conference on Intelligent Computation Technology and Automation (ICICTA). Vol. 2, pp. 674-678.

PEGDEN, C. D. 2007. Simio: A new simulation system based on intelligent objects. Proceedings of the 39th Winter Simulation Conference: 40 years! The best is yet to come, pp. 2293-2300, IEEE Press.

PEGDEN, C. D. \& STURROCK, D. T. 2008. Introduction to Simio. 2008 Winter Simulation Conference, pp 29-38, IEEE.

SKRODENIS, E., VINGRYS, S. \& PASHKEVICH, M. 2011. Lithuanian experience of implementation of roundabouts: the research of accidents, operation and efficiency. The 8th International Conference "Environmental Engineering": Selected papers. Ed. by D. Čygas, KD Froehner, pp. 980-985. 
STURROCK, D. T. \& PEGDEN, C. D. 2010. Recent innovations in Simio. Proceedings - Winter Simulation Conference, pp. 52-62. Proceedings - Winter Simulation Conference, pp. 52-62.

TONG, H. Y., HUNG, W. T. \& CHEUNG, C. S. 2000. On-Road Motor Vehicle Emissions and Fuel Consumption in Urban Driving Conditions. Journal of the Air \& Waste Management Association, 50, 543-554.

TREIBER, M. \& HELBING, D. 2001. Microsimulations of Freeway Traffic Including Control Measures. at - Automatisierungstechnik, 49, 478.

VIEIRA, A., DIAS, L., PEREIRA, G. \& OLIVEIRA, J. 2014a. Comparison of Simio and Arena simulation tools. ISC. University of Skovde, Skovde, Sweden.

VIEIRA, A., DIAS, L., PEREIRA, G. \& OLIVEIRA, J. 2014b. Micro Simulation to Evaluate the Impact of Introducing Pre-Signals in Traffic Intersections. University of Minho at Guimarães - Portugal: International Conference on Computational Science and its Application, pp. 722-745, Springer International Publishing.

WU, J. \& HOUNSELL, N. 1998. Bus Priority Using pre-signals. Transportation Research Part A: Policy and Practice, 32, 563-583.

XIE, H. \& MA, W. 2012. Simulation-based study on a pre-signal control system at isolated intersection with separate left turn phase. 9th IEEE International Conference on Networking, Sensing and Control (ICNSC), pp. 103-106.

XUAN, Y., DAGANZO, C. F. \& CASSIDY, M. J. 2011. Increasing the capacity of signalized intersections with separate left turn phases. Transportation Research Part B: Methodological, 45, 769-781.

ZEGEER, J. D. 1986. Field Validation of Intersection Capacity Factor. Transportation Research Record 1091, pp.67-77.

ZHOU, Y. \& ZHUANG, H. 2013. The optimization of lane assignment and signal timing at the tandem intersection with pre-signal. Journal of Advanced Transportation, 48(4), pp. 362-376.

ZHU, H. 2008. Normal Acceleration Characteristics of the Leading Vehicle in a Queue at Signalized Intersections on Arterial Streets, Doctoral dissertation. Oregon State University. 